\title{
Effect of genetic variation in UGT1A and $A B C B 1$ on moxifloxacin pharmacokinetics in South African patients with tuberculosis
}

\author{
Anushka Naidoo*,1, Veron Ramsuran ${ }^{1,2}$, Maxwell Chirehwa ${ }^{3}$, Paolo Denti $^{3}$, Helen \\ Mcllleron $^{3}$, Kogieleum Naidoo ${ }^{1,4}$, Nonhlanhla Yende-Zuma ${ }^{1}$, Ravesh Singh ${ }^{5}$, Sinaye \\ Ngcapu ${ }^{1}$, Mamoonah Chaudhry ${ }^{6}$, Michael S Pepper ${ }^{6}$ \& Nesri Padayatchi ${ }^{1,4}$ \\ ${ }^{1}$ Centre for the AIDS Programme of Research in South Africa (CAPRISA), University of KwaZulu-Natal, Durban, KwaZulu-Natal, \\ South Africa \\ ${ }^{2}$ School of Laboratory Medicine \& Medical Sciences, University of KwaZulu-Natal, Durban, KwaZulu-Natal, South Africa \\ ${ }^{3}$ Department of Medicine, Division of Clinical Pharmacology, University of Cape Town, Western Cape, South Africa \\ ${ }^{4}$ MRC-CAPRISA HIV-TB Pathogenesis \& Treatment Research Unit, Doris Duke Medical Research Institute, University of \\ KwaZulu-Natal, KwaZulu-Natal, South Africa \\ ${ }^{5}$ Department of Microbiology, National Health Laboratory Services, KZN Academic Complex, Inkosi Albert Luthuli Central Hospital, \\ Durban, KwaZulu-Natal, South Africa \\ ${ }^{6}$ Department of Immunology \& the Institute for Cellular \& Molecular Medicine; South African Medical Research Council Extramural \\ Unit for Stem Cell Research \& Therapy, Faculty of Health Sciences, University of Pretoria, Pretoria, Gauteng, South Africa \\ * Author for correspondence: Tel.: +27 31260 1963; anushka.naidoo@caprisa.org
}

\begin{abstract}
Aim: We assessed the effect of genetic variability in UGT1A and $A B C B 1$ genes on moxifloxacin pharmacokinetics. Methods: Genotypes for selected UGT1A and $A B C B 1$ SNPs were determined using a TaqMan ${ }^{\circledR}$ Genotyping OpenArray ${ }^{\mathrm{TM}}$ and high-resolution melt analysis for rs8175347. A nonlinear mixed-effects model was used to describe moxifloxacin pharmacokinetics. Results: Genotypes of UGT1A SNPs, rs8175347 and rs3755319 (20.6\% lower and $11.6 \%$ increased clearance, respectively) and $A B C B 1$ SNP rs 2032582 (40\% reduced bioavailability in one individual) were significantly associated with changes in moxifloxacin pharmacokinetic parameters. Conclusion: Genetic variation in UGT1A as represented by rs 8175347 to a lesser extent rs3755319 and the $A B C B 1$ rs2032582 SNP is modestly associated with the interindividual variability reported in moxifloxacin pharmacokinetics and exposure. Clinical relevance of the effects of genetic variation on moxifloxacin pharmacokinetic requires further investigation.
\end{abstract}

First draft submitted: 17 August 2017; Accepted for publication: 10 October 2017; Published online: 6 December 2017

Keywords: $A B C B 1 \bullet$ moxifloxacin $\bullet$ pharmacogenetics $\bullet$ pharmacokinetics $\bullet$ UGT1A

Moxifloxacin, an 8-methoxy fluoroquinolone, is active against both Gram-positive and Gram-negative bacteria, including anaerobes, and is used to treat a number of bacterial infections [1]. Moxifloxacin is also used in the treatment of Mycobacterium tuberculosis. It is recommended by the WHO for the treatment of multidrug-resistant tuberculosis (MDR-TB) and for drug-susceptible TB if toxicity develops to standard first-line drugs or for isoniazid (INH) monoresistance [2]. Moxifloxacin is emerging as a key drug in the development of novel shorter rifampicinsparing drug regimens for the treatment of both drug susceptible and MDR-TB, being investigated in several Phase III clinical trials, which are currently underway [3,4].

Although moxifloxacin demonstrates potent bactericidal activity in the treatment of tuberculosis, evidence suggests that the current standard $400 \mathrm{mg}$ dose may not be optimal in all patients, resulting in poor treatment outcomes and increased risk of acquired drug resistance [5]. Moxifloxacin exhibits extensive interpatient pharmacokinetic (PK) variability with widely ranging $C_{\max }$ and area under the curve (AUC) in both healthy individuals and patients with TB [6-10]. The observed variability in pharmacokinetics may be due to several factors including differences in patient age, weight, disease status, gender, poor treatment adherence, co-morbidities such as HIV and drug interactions [11]. In addition, genetic variation associated with SNPs, copy number variants and insertions or deletions 
in genes coding for drug metabolizing and transporter enzymes is emerging as an important contributing factor to TB drug exposure and variable PK parameters [12].

African populations have high levels of host genetic diversity resulting in differences in TB disease susceptibility [13]. Furthermore, genetic diversity in drug metabolizing and drug transport enzymes has been shown to result in lower TB drug concentrations and variations in response to standard first-line TB drugs [14-16].

Moxifloxacin is metabolized via glucuronide and sulfate conjugation by cytosolic enzymes glucuronosyltransferase and sulfotransferase [17]. The UGT's form a family of enzymes, of which the UGT1A subfamily is encoded by a single gene locus through differential splicing of unique first exons (exon 1) to shared exons 2-5. The major human UGT's responsible for formation of the moxifloxacin M2 metabolite are UGT1A1 (main isoform), UGT1A3 and UGT1A9. Although there is no previous published data on polymorphisms in the genes encoding for UGT enzymes, which might affect moxifloxacin metabolism or pharmacokinetics in TB treatment, these enzymes are known to be highly polymorphic leading to altered concentrations of other drugs and of moxifloxacin in healthy individuals [18-20]. Moxifloxacin is a substrate of the multidrug transporter P-glycoprotein, and this drug transporter protein found in the liver, kidneys and intestinal mucosa plays an important role in its absorption distribution and elimination [21,22]. P-glycoprotein is coded by the $M D R 1$ or $A B C B 1$ gene, which forms part of the ABCB1 family and is located on chromosome 7q21.12, spanning $209.6 \mathrm{~kb}$ and containing 29 exons [23]. Limited data from one study investigating polymorphisms in the $A B C B 1$ (MDR1 C3435T) gene coding for this transport protein found that the MDR1 $3435 C C$ variant may affect the absorption of moxifloxacin in healthy individuals [8]. $A B C B 1$ exhibits high functional variance [24] shown to affect other drug exposures [23]. The effects of polymorphisms in $A B C B 1$ and genes coding for UGT enzymes on moxifloxacin pharmacokinetics in patients with TB need further investigation $[18,24]$.

The purpose of this study was to determine the prevalence of genetic variability in $U G T 1 A$ and $A B C B 1$ in a cohort of South African patients with drug-susceptible recurrent TB, and to assess whether this variability might impact on moxifloxacin pharmacokinetics and drug exposure.

\section{Methods}

A prospective PK substudy was conducted within the ongoing Improving Retreatment Success (IMPRESS) openlabel randomized controlled trial (NCT02114684) from October 2013 in KwaZulu-Natal, Durban, South Africa. Details on study objectives, design and inclusion or exclusion criteria are available at ClinicalTrials.gov (https: //clinicaltrials.gov/ct2/show/NCT02114684).

Patients in the intervention arm of the study receiving moxifloxacin, who provided informed consent to be included in the PK substudy, had blood samples collected for PK analysis at predefined time points. Baseline whole blood samples were collected in all patients consenting to storage of samples for later genetic testing. All participants recruited to the study were: $>18$ years of age, had a confirmed history of TB within the last 3 years and had been diagnosed with sputum smear positive, rifampicin sensitive, $M$. tuberculosis based on microscopy and GeneXpert technology. Only those with no predefined laboratory or clinical abnormalities were included, regardless of HIV status.

\section{Drug regimens}

Patients randomized to the intervention arm of the study received daily moxifloxacin $400 \mathrm{mg}$ (Avelox ${ }^{\circledR}$ - Bayer Healthcare, Leverkusen, Germany), weight-based rifampicin 450 or $600 \mathrm{mg}$ and isoniazid 225 or $300 \mathrm{mg}$, for patients $38-54 \mathrm{~kg}$ and $\geq 55 \mathrm{~kg}$, respectively. Rifampicin, isoniazid and moxifloxacin were included during the 2-month intensive phase and 4-month continuation phase of TB treatment. Pyrazinamide 1500 and $2000 \mathrm{mg}$, in patients between $38-54 \mathrm{~kg}$ and $\geq 55 \mathrm{~kg}$, respectively, was used during the intensive phase of treatment.

\section{Follow-up}

Patients were followed up for 24 months, and clinical and safety monitoring was done bimonthly for the first 6 months, or as clinically indicated. Adherence to TB treatment was measured using pill count based on the number of tablets dispensed, physically returned, reported remaining or lost as well as participant self-report of missed or incomplete doses in the 4 days prior to the day of study visit or PK sampling. HIV co-infected patients received standard first-line antiretroviral therapy (ART) containing efavirenz, emtricitabine and tenofovir. Treatment and prophylaxis for opportunistic infections and concomitant treatment used were recorded on case report forms. 


\section{PK sample collection}

Plasma samples were collected prior to drug dose and at 2.5, 6 and $24 \mathrm{~h}$ post dose at month 1 and/or 2 during the intensive phase of TB treatment, at month 6 during the continuation phase of TB treatment and at approximately 4 weeks after completion of TB treatment following a single dose of moxifloxacin. Plasma, collected in ethylenediaminetetraacetic acid (EDTA) tubes, was centrifuged at 3000 r.p.m., placed on ice, sent within $1 \mathrm{~h}$ of collection to the CAPRISA laboratory to be stored at $-80^{\circ} \mathrm{C}$. Moxifloxacin concentrations were quantified in clinical plasma samples at the KwaZulu-Natal Research Institute for tuberculosis and HIV (KRITH) pharmacology laboratory using validated HPLC and tandem mass spectrometry. The bioanalytical method was developed and validated according to US FDA guidelines (2011) [25]. Sample preparation included a protein precipitation with acetonitrile and subsequent dilution with water. Analytes were chromatographically separated using a Zorbax C18, $3.5 \mu \mathrm{m}, 50 \mathrm{~mm} \times 2.1 \mathrm{~mm}$ column and detected using the ABI Sciex 5500 (AB Sciex LLC, MA, USA). QTrap mass spectrometer (AB Sciex LLC) operated in positive mode. The following transitions were used: precursor ion $\rightarrow$ product ion (all in units of $\mathrm{m} / \mathrm{z}$ ): moxifloxacin: $402.1 \rightarrow 358.2$ and $402.1 \rightarrow 364.1$. The internal standard used was ciprofloxacin: $331.6 \rightarrow 231.0$ and $331.6 \rightarrow 288.1$. Moxifloxacin was analyzed isocratically with a $22 \%$ acetonitrile/water $/ 0.1 \%$ formic acid mobile phase. The injection volume was $2 \mu \mathrm{l}$ and the total analytical run time was $5 \mathrm{~min}$. The method was validated over the concentration range of 50-5000 ng/ml. Overall precision, based on quality control samples evaluated at low, medium and high concentrations during the validation and analysis of samples ranged from 8.4 to $19.4 \%$ and accuracy ranged from 101.9 to $105 \%$. Calculated carry over at the lower limit of quantification was $5.4 \%$. The liquid chromatography-tandem mass spectrometry system was interfaced with a DELL ${ }^{\circledR}$ Windows ${ }^{\circledR} 7$ computer running Analyst ${ }^{\circledR}$ software version 1.6.2, which was used for chromatographic data acquisition, peak integration and quantification of analytes.

\section{Genotyping}

A total of 14 SNPs, six for $A B C B 1$ and eight for $U G T 1 A$, were selected for analysis in this study based on previous evidence of functional significance on drug response using the PharmGKB database and relevant literature [18,24,26]. The SNPs selected included rs10276036, rs1128503, rs2032582, rs1045642, rs2235033 and rs2235013 for $A B C B 1$; and rs4148323, rs2003569, rs3755319, rs11692021, rs2070959, rs28900377, rs1983023 and rs8175347 for $U G T 1 A$.

Genotypes were determined for each of the SNPs using a TaqMan ${ }^{\circledR}$ Genotyping OpenArray ${ }^{\mathrm{TM}}$ (Thermo Fisher Scientific, MA, USA). For rs8175347, due to the limited ability of TaqMan probes to reliably distinguish all genotypes, a high resolution melt analysis was used to determine genotype [27]. The TaqMan Genotyping OpenArray was performed as follows: genomic DNA was extracted from whole blood for each sample using the Qiagen QIAamp MiniKit (Germany, cat. no. 51306) according to manufacturer's instructions. Approximately $50 \mu \mathrm{g} / \mathrm{ml}$ of DNA and $2 \times$ TaqMan Genotyping OpenArray Master Mix was used for the assay. Plates were loaded using the Accufill liquid handling robot and run on the Applied Biosystems ${ }^{\mathrm{TM}}$ QuantStudio $^{\mathrm{TM}} 12 \mathrm{~K}$ Flex Real-Time PCR instrument according to Applied Biosystems ${ }^{\mathrm{TM}}$ (CA, USA) user guide. Analysis was performed using the TaqMan Genotyper Software version 3.1. The genotypes for UGT1A1*28 (rs8175347) were determined using genomic DNA as previously described [27] with minor modifications. The real-time PCR conditions were changed as follows: $95^{\circ} \mathrm{C}$ for 5 mins, followed by 45 cycles of $95^{\circ} \mathrm{C}$ for $10 \mathrm{~s}, 67^{\circ} \mathrm{C}$ for $10 \mathrm{~s}$ and $76^{\circ} \mathrm{C}$ for $10 \mathrm{~s}$ and finally $76^{\circ} \mathrm{C}$ for $1 \mathrm{~min}$. Linkage disequilibrium (LD) was tested and haplotypes generated using LDlink 2.0. online software [28]. SNPs from UGT1A (rs11692021 and rs2070959) were found to be in perfect $\mathrm{LD}\left(\mathrm{D}^{\prime}=1, \mathrm{r}^{2}=1\right)$. In addition, $A B C B 1$ SNPs (rs2235033 and rs2235013) were found to be in perfect $\mathrm{LD}\left(\mathrm{D}^{\prime}=1, \mathrm{r}^{2}=1\right)$. The rs11692021 and rs2235033 SNPs were randomly selected in order to exclude them from the haplotypes generated.

\section{Study oversight}

Ethical approval for the study was provided by the Biomedical Research Ethics Committee of the University of KwaZulu-Natal (BFC029/13) and the Medicines Control Council of South Africa (MCC ref:20130510).

\section{Statistical analysis}

The moxifloxacin concentration-time data were analyzed using nonlinear mixed-effects (NLME) modelling, implemented with the software NONMEM (version 7.3) [29]. Perl-speaks-NONMEM, Xpose and Pirana were used for model diagnostics and to track model development [30]. A previously reported model developed on the same PK data was used as a starting point [31]. The model had a semimechanistic structure describing the effect of the liver 
Table 1. Baseline data.

\begin{tabular}{|c|c|c|}
\hline Variable & $n=172$ & $n=58$ \\
\hline Age (years), median (IQR) & $35(30-41)$ & $37(31-42)$ \\
\hline Male, n (\%) & $119(69.2)$ & $41(70.7)$ \\
\hline $\begin{array}{l}\text { Race, n (\%) - Black African } \\
\text { ethnicity/Caucasian/colored }\end{array}$ & $170(98.8) / 1(0.6) / 1(0.6)$ & $56(96.6) / 1(1.7) / 1(1.7)$ \\
\hline Weight (kg), median (IQR) & $55.7(50.3-62.1)$ & $56.9(51.1-65.2)$ \\
\hline Fat-free mass $(\mathrm{kg})$ & - & $46.8(42.5-50.3)$ \\
\hline BMI $\left(\mathrm{kg} / \mathrm{m}^{2}\right)$, median (IQR) & $19.7(18.3-22.5)$ & $19.6(18.0-23.3)$ \\
\hline HIV status, $\mathrm{n}(\%)$ positive/negative & $127(73.8) / 45(26.2)$ & $42(72.4) / 16(27.6)$ \\
\hline \multicolumn{3}{|l|}{ ART, n (\%) } \\
\hline Efavirenz + Emtricitabine + Tenofovir & $117(95.1)$ & $40(95.2)$ \\
\hline Lopinavir/Ritonavir + Lamivudine + Tenofovir & $2(1.6)$ & $2(4.8)$ \\
\hline Lopinavir/Ritonavir + Lamivudine + Zidovudine & $2(1.6)$ & - \\
\hline Lopinavir/Ritonavir + Emtricitabine + Tenofovir & $1(0.8)$ & - \\
\hline Efavirenz/Lamivudine/Zidovudine & $1(0.8)$ & - \\
\hline $\mathrm{CD}^{+}$count (cells $\left./ \mathrm{mm}^{3}\right)$, median $(\mathrm{IQR})^{\dagger} \S$ & $241.0(129.0-407.0)$ & $277.0(139.0-384.0)$ \\
\hline Viral load (log 10 copies/ml) & $3.7(1.3-5.0)$ & $3.3(1.3-4.2)$ \\
\hline $\begin{array}{l}{ }^{\dagger} \text { Mixed race. } \\
\text { ‡Only for HIV+ patients. } \\
\S 10 / 172,4 / 58 \text { missing data. } \\
\text { \$12/172, 5/58 missing data. } \\
\text { ART: Antiretroviral therapy; IQR: Interquartile range. }\end{array}$ & & \\
\hline
\end{tabular}

both on first-pass extraction and systemic clearance. The original analysis [31] identified and characterized the effect of several factors on the pharmacokinetics of moxifloxacin, including body size (using fat-free mass of the individual patients, calculated based on weight, height and sex as suggested by Janmahasatian et al. [32]), co-administration of efavirenz and a difference between the exposure obtained at steady-state during rifampicin-based TB treatment and at single dose. After accounting for these effects, a moderate amount of variability still remained in the data, and this analysis aimed to further improve the model by exploring and including the effect of genetic polymorphisms. As a first exploratory step, random effects for the between-subject and -occasion variability in intrinsic clearance $\left(\mathrm{CL}_{\text {int }}\right)$, prehepatic bioavailability and absorption parameters were extracted from the original model. These represent the portion of variability not yet explained by the factors previously included in the model (mentioned above). Linear mixed-effects models implemented in SAS, version 9.4 (SAS Institute, NC, USA) were used to explore the relationship between this variability and genotypes for the $U G T 1 A$ and $A B C B 1$ SNPs. A compound symmetry correlation structure was assumed to describe the correlation between individual variability estimates at different visits. After this screening step, all the effects for which a trend was identified $(p<0.05)$ were further assessed within an updated version of the NLME model using stepwise covariate model building implemented in Perl-speaks-NONMEM [30]. Each SNP was included as a categorical variable, and grouped according to the corresponding genotype - that is, wild-type, heterozygous, or homozygous mutant. In certain instances, the heterozygous group was combined with either the wild-type or homozygous mutant whenever no statistical significant difference could be detected between two of the variants. A drop in the NONMEM objective function value (OFV) of 3.84 (corresponding $5 \%$ level of significance, 1 degree of freedom) was considered for inclusion of the effect of an SNP. A more stringent criterion of a change in OFV of 6.64 (corresponding to $1 \%$ level of significance) was applied for retaining the effect of an SNP in the final model. Further diagnostics were used, including inspection of goodness of fit plots and visual predictive checks, testing the robustness of the detected effect with a nonparametric bootstrap with replacement $(\mathrm{n}=200)$, and inspection of the individual contributions to the change of the OFV.

\section{Results}

Baseline data

We included 172 South African TB patients: 119 (69.2\%) male, 170 (98.8\%) of Black African ethnicity and 127 (73.8\%) HIV co-infected (Table 1). Moxifloxacin concentration-time data were available for 58 of 172 patients. Of these: median weight, fat-free mass and age were $56.9 \mathrm{~kg}$ (interquartile range [IQR]: $51.1-65.2), 46.8 \mathrm{~kg}$ (IQR: 
42.5-50.3) and 37 years (IQR: 31-42), respectively. Forty-one (70.7\%) patients were male, 42 (72.4\%) HIV co-infected, with 40/42 (95\%) on efavirenz-based ART (Table 1).

\section{Genotype frequencies}

The genotypic frequencies for $U G T 1 A$ and $A B C B 1$ are presented in Table 2 for all patients $(\mathrm{n}=172)$ included in the study and the subset of 58 patients with moxifloxacin drug level data. Allelic frequencies for all SNPs are presented in Table 3. Haplotypes were created for UGT1A based on SNPs rs2070959, rs1983023, rs28900377, rs3755319, rs2003569 and rs4148323 while haplotypes for $A B C B 1$ were created based on SNPs rs1128503, rs2032582 and rs 1045642, in the order listed. Haplotype frequencies are presented in Table 2.

\section{Effects of UGT1A and $A B C B 1$ SNPs on moxifloxacin pharmacokinetics}

The variability in mean moxifloxacin plasma concentrations (AUC) in each patient obtained from the base NLME model and stratified by genotypes for eight $U G T 1 A$ and six ABCB1 SNPs are shown in Figure 1A and $\mathrm{B}$. These values are affected by other effects contained in the base model, and are only reported to show the distribution of variability in AUC among genotypes tested and for comparison of exposures with other studies. The exploration of pharmacogenetics was executed after adjusting for all the fixed effects already included in the model.

An LD test was performed on UGT1A and ABCB1 SNPs (Figure 1C \& D). For UGT1A we tested seven SNPs. Of these, rs 11692021 and rs2070959 were found to be in perfect $L D\left(D^{\prime}=1, r^{2}=1\right)$. For this reason, rs 11692021 was randomly selected and excluded from the generation of haplotypes. The LD was not calculated for rs 4148323 because only one genotype (GG) was observed in the population (Figure 1C). LD analysis was performed on five SNPs for $A B C B 1$; rs2032582 was not included in the LD analysis as this SNP possessed multiple alleles that did not fit within the LD test. We found rs2235033 and rs2235013 to be in perfect $L D\left(D^{\prime}=1, r^{2}=1\right.$; Figure $\left.1 D\right)$; rs2235033 was randomly chosen to be excluded from the generation of haplotypes.

The SNPs found to be significant in the screening step employing linear models were then tested in NLME models that allow for re-estimation of other covariates that may affect moxifloxacin PK parameters. The effects of two UGT SNPs were confirmed to influence the pharmacokinetics of moxifloxacin in the final NLME PK model (Table 4). Having the TA $5 / 6$ repeat in rs 8175347 was associated with a $20.6 \%$ lower clearance and approximately $26 \%$ higher AUC, after adjusting for other covariates (such as efavirenz and/or rifampicin co-administration) compared with those with TA $6 / 6,6 / 7,7 / 7$ and $7 / 8(\mathrm{p}=0.001)$. Patients with AC and AA genotypes for rs3755319 were found to have $11.6 \%$ increased clearance when compared with the CC genotype in the model $(p=0.032)$. However, effect of the SNP was not strong enough to be retained in the final model using the cut-off value of $\mathrm{p}=0.01$. Only one individual within the cohort examined had a CA genotype for the $A B C B 1$ SNP rs2032582. When the effect of the rs2032582 SNP was included in the population PK model, a $40 \%$ reduced prehepatic bioavailability and similar decrease in AUC were observed for the patient with the CA genotype $(p=0.01)$. However, although this effect significantly improved the model fit in terms of OFV, it was not retained in the final model due to the fact that only one patient was affected. When testing the outcome of presence versus absence of haplotypes for both $A B C B 1$ and $U G T$ within individual patients in the NLME models, no significant difference was observed. Figure 2 depicts the visual predictive checks for the final model stratified by genotypes for rs8175347. The visual predictive checks show that the model describes the data adequately after inclusion of the effect of the SNP.

\section{Discussion}

We describe the prevalence of genetic variability in selected UGT1A and ABCB1 SNPs in a cohort of South African TB patients, the majority being of Black African ethnicity. Our data add to the current evidence of genetic variants having pharmacogenetic relevance among Africans. The impact of genetic variation on drug metabolizing and transporter enzymes UGT and ABCB1, has not been described previously for moxifloxacin in the context of TB treatment. We found differences between genotypes of UGT1A SNPs rs8175347 and rs3755319, which significantly associated with changes in moxifloxacin PK parameters. Genotypes of the $A B C B 1$ SNP rs2032582 were significantly associated with a decrease in prehepatic bioavailability in the NLME model.

Moxifloxacin exhibits extensive interindividual variability in PK parameters [6-8,33,34]. We tested the effect of genetic variants in $U G T 1 A$ and $A B C B 1$ as covariates in NLME models used to determine moxifloxacin concentrationtime data and found that these are associated with some of the between subject variability observed in moxifloxacin PK parameters not accounted for by other relevant covariates in our study. We previously reported low overall 
Research Article Naidoo, Ramsuran, Chirehwa et al.

Table 2. Genotype and haplotype frequency for UGT1A and ABCB1 SNPs.

\begin{tabular}{|c|c|c|c|c|c|c|c|}
\hline \multicolumn{4}{|c|}{ Frequency UGT1A } & \multicolumn{4}{|c|}{ Frequency $A B C B 1$} \\
\hline SNP & Genotype & $n=172$ & $n=58$ & SNP & Genotype & $n=172$ & $n=58$ \\
\hline \multirow[t]{3}{*}{$\begin{array}{l}\text { rs11692021 } \\
622 T>C\end{array}$} & $\mathrm{CC}$ & 0.04 & 0.05 & $\begin{array}{l}r s 10276036 \\
1000-44 G>A\end{array}$ & $\mathrm{TT}$ & 0.74 & 0.74 \\
\hline & $\mathrm{CT}$ & 0.18 & 0.21 & & $\mathrm{CT}$ & 0.23 & 0.24 \\
\hline & $\mathrm{TT}$ & 0.78 & 0.73 & & $\mathrm{CC}$ & 0.03 & 0.02 \\
\hline \multirow[t]{3}{*}{ rs4148323 211G >A } & GG & 1.00 & 1.00 & $\begin{array}{l}\mathrm{rs} 1128503 \\
1236 \mathrm{C}>\mathrm{T}\end{array}$ & AA & 0.01 & 0.00 \\
\hline & GA & 0.00 & 0.00 & & AG & 0.14 & 0.16 \\
\hline & AA & 0.00 & 0.00 & & GG & 0.85 & 0.84 \\
\hline \multirow[t]{3}{*}{$\begin{array}{l}r s 2003569 \\
-997 G>A\end{array}$} & AA & 0.18 & 0.09 & $\begin{array}{l}\mathrm{rs} 2032582 \\
2677 \mathrm{G}>\mathrm{T} / \mathrm{A}\end{array}$ & AA & 0.00 & 0.00 \\
\hline & AG & 0.38 & 0.40 & & CA & 0.02 & 0.03 \\
\hline & GG & 0.45 & 0.51 & & $\mathrm{CC}$ & 0.98 & 0.97 \\
\hline \multirow[t]{3}{*}{$\begin{array}{l}\text { rs3755319 } \\
-1352 A>C\end{array}$} & $\mathrm{AA}$ & 0.06 & 0.05 & $\begin{array}{l}\mathrm{rs} 1045642 \\
3435 \mathrm{C}>\mathrm{T}\end{array}$ & AA & 0.02 & 0.00 \\
\hline & $A C$ & 0.32 & 0.41 & & AG & 0.14 & 0.19 \\
\hline & $\mathrm{CC}$ & 0.62 & 0.54 & & GG & 0.84 & 0.81 \\
\hline \multirow[t]{3}{*}{$\begin{array}{l}\text { rs } 2070959 \\
855+20756 A>G\end{array}$} & AA & 0.79 & 0.74 & $\begin{array}{l}r s 2235013 \\
1725+38 G>A\end{array}$ & $\mathrm{TT}$ & 0.29 & 0.38 \\
\hline & AG & 0.18 & 0.21 & & $\mathrm{CT}$ & 0.47 & 0.43 \\
\hline & GG & 0.04 & 0.05 & & $\mathrm{CC}$ & 0.24 & 0.20 \\
\hline \multirow[t]{3}{*}{$\begin{array}{l}r s 28900377 \\
856-23423 C>T\end{array}$} & $\mathrm{TT}$ & 0.01 & 0.00 & $\begin{array}{l}r s 2235033 \\
1554+24 T>C\end{array}$ & AA & 0.24 & 0.20 \\
\hline & $\mathrm{CT}$ & 0.06 & 0.09 & & AG & 0.76 & 0.80 \\
\hline & $\mathrm{CC}$ & 0.93 & 0.91 & & GG & 0.00 & 0.00 \\
\hline \multirow{3}{*}{$\begin{array}{l}\text { rs1983023 } \\
-751 T>C\end{array}$} & $\mathrm{TT}$ & 0.06 & 0.07 & & & & \\
\hline & $\mathrm{CT}$ & 0.39 & 0.36 & & & & \\
\hline & $\mathrm{CC}$ & 0.56 & 0.57 & & & & \\
\hline \multirow[t]{8}{*}{ rs8175347 TA [5-8] } & $\begin{array}{l}\text { TA5 } / 6- \\
\text { UGT1A } 1 * 36 / * 1\end{array}$ & 0.11 & 0.10 & & & & \\
\hline & $\begin{array}{l}\text { TA5 } / 7- \\
\text { UGT1A1*36/*28 }\end{array}$ & 0.01 & 0.00 & & & & \\
\hline & $\begin{array}{l}\text { TA5 } / 8- \\
\text { UGT1A1*36/*37 }\end{array}$ & 0.01 & 0.00 & & & & \\
\hline & $\begin{array}{l}\text { TA6/6 - } \\
\text { UGT1A } 1 * 1 / * 1\end{array}$ & 0.48 & 0.50 & & & & \\
\hline & $\begin{array}{l}\text { TA6/7 - } \\
\text { UGT1A1*1/*28 }\end{array}$ & 0.16 & 0.12 & & & & \\
\hline & $\begin{array}{l}\text { TA6/8 - } \\
\text { UGT1A1*1/*37 }\end{array}$ & 0.02 & 0.02 & & & & \\
\hline & $\begin{array}{l}\text { TA7 } / 7- \\
\text { UGT1A1*28/*28 }\end{array}$ & 0.08 & 0.12 & & & & \\
\hline & $\begin{array}{l}\text { TA7 } / 8- \\
\text { UGT1A1*28/*37 }\end{array}$ & 0.13 & 0.14 & & & & \\
\hline UGT1A haplotype & & $n=172$ & $n=58$ & $A B C B 1$ & Haplotype & $\mathrm{n}=172$ & $n=58$ \\
\hline Hap1 & ACCCGG & 0.71 & 0.76 & & CGC & 0.83 & 0.84 \\
\hline Hap2 & GCCCGG & 0.20 & 0.24 & & CGT & 0.15 & 0.20 \\
\hline Hap3 & ACCAGG & 0.35 & 0.40 & & CTC & 0.02 & 0.02 \\
\hline Hap4 & ACCCAG & 0.51 & 0.44 & & TGC & 0.15 & 0.16 \\
\hline Hap5 & ATCAGG & 0.24 & 0.25 & & TGT & 0.10 & 0.14 \\
\hline Hap6 & ATCCAG & 0.27 & 0.22 & & & & \\
\hline Hap7 & ATTCAG & 0.05 & 0.05 & & & & \\
\hline Hap8 & GCTCGG & 0.01 & 0.04 & & & & \\
\hline
\end{tabular}


Table 3. Allele frequencies for UGT1A and ABCB1 SNPs $(n=172)$. UGT1A

\begin{tabular}{|c|c|c|c|c|c|c|c|}
\hline $211 \mathrm{G}>\mathrm{A}$ & $-997 \mathrm{G}>\mathrm{A}$ & $-1352 A>C$ & $855+20756 A>G$ & $856-23423 \mathrm{C}>\mathrm{T}$ & $-751 \mathrm{~T}>\mathrm{C}$ & $\mathrm{TA}_{(\mathrm{n})}$ & $622 \mathrm{~T}>\mathrm{C}$ \\
\hline rs4148323 & rs 2003569 & rs 3755319 & rs 2070959 & rs28900377 & rs 1983023 & rs 8175347 & rs11692021 \\
\hline G (1.00) & $A(0.36)$ & $A(0.22)$ & $\mathrm{A}(0.87)$ & $\mathrm{T}(0.04)$ & $\mathrm{T}(0.25)$ & $5(0.07)$ & $C(0.13)$ \\
\hline \multirow{2}{*}{$A(0.00)$} & & & & & & $7(0.23)$ & \\
\hline & & & & & & $8(0.08)$ & \\
\hline \multicolumn{8}{|l|}{$A B C B 1^{\dagger}$} \\
\hline rs10276036 & rs1128503 & rs2032582 & rs1045642 & rs2235013 & rs2235033 & & \\
\hline $\mathrm{T}(0.85)$ & $A(0.08)$ & $A(0.01)$ & $A(0.09)$ & $\mathrm{T}(0.53)$ & $\mathrm{A}(0.47)$ & & \\
\hline$C(0.15)$ & $\mathrm{G}(0.92)$ & $C(0.99)$ & $\mathrm{G}(0.91)$ & $C(0.47)$ & G (0.53) & & \\
\hline
\end{tabular}

†The genotypes represented for $A B C B 1$ represent the forward orientation while the gene is transcribed in the reverse direction. $\mathrm{TA}_{(\mathrm{n})}$ : number of TA repeats.

Table 4. Population parameter estimates of moxifloxacin pharmacokinetics.

\begin{tabular}{|c|c|c|}
\hline Parameter description & Typical value $(\mathrm{Cl})^{\S}$ & Random variability $(\mathrm{Cl})^{5}$ \\
\hline $\begin{array}{l}\text { Intrinsic clearance during rifampicin-based TB } \\
\text { treatment }(I / h)^{\dagger, \ddagger}\end{array}$ & $50.0(45.9 ; 56.8)$ & $\begin{array}{l}\text { BSV: } 12.1 \%(2.6 ; 16.7) \\
\text { BOV: } 12.2 \%(4.6 ; 18.9)\end{array}$ \\
\hline Volume of central compartment $(\mathrm{I})^{\ddagger}$ & $127(109 ; 137)$ & BSV: $8.6 \%(0.5 ; 13.5)$ \\
\hline Intercompartmental clearance $(\mathrm{I} / \mathrm{h})^{\ddagger}$ & $2.12(1.55 ; 4.51)$ & - \\
\hline Volume of peripheral compartment $(\mathrm{I})^{\ddagger}$ & $31.0(22.3 ; 50.8)$ & - \\
\hline Prehepatic bioavailability (.)\# & 1 fixed & BOV: $35.6 \%(28.2 ; 42.1)$ \\
\hline Absorption lag time $(h)^{\text {prior }}$ & $0.55(0.45 ; 0.73)$ & - \\
\hline Ka - absorption rate $(1 / h)^{\text {prior }}$ & $2.80(1.18 ; 3.45)$ & BOV: $93.2 \%(0.9 ; 121.7)$ \\
\hline Hepatic plasma flow $(\mathrm{l} / \mathrm{h})$ & 50 fixed & \\
\hline Moxifloxacin fraction unbound (\%) & $50 \%$ fixed & \\
\hline $\begin{array}{l}\text { Change in intrinsic clearance while on single dose of } \\
\text { moxifloxacin (\%) }\end{array}$ & $-28.9 \%(-36.5 ;-21.9)$ & \\
\hline $\begin{array}{l}\text { Change in prehepatic bioavailability while on single } \\
\text { dose of moxifloxacin (\%) }\end{array}$ & $-22.4 \%(-32.4 ;-11.6)$ & \\
\hline $\begin{array}{l}\text { Change in intrinsic clearance while on efavirenz-based } \\
\text { ART }(\%)\end{array}$ & $+42.9 \%(32.6 ; 56.1)$ & \\
\hline $\begin{array}{l}\text { Change in intrinsic clearance due to rs } 8175347 \text { (five } \\
\text { out of six genotypes) }\end{array}$ & $-20.6 \%(-29.3 ;-13.6)$ & \\
\hline $\begin{array}{l}\text { Scaling factor for variability in bioavailability while on } \\
\text { single dose of moxifloxacin (-fold) }\end{array}$ & 0.62 -fold $(0.41 ; 0.85)$ & \\
\hline $\begin{array}{l}\text { Scaling factor for variability in bioavailability for } \\
\text { unobserved doses (-fold) })^{\dagger \dagger}\end{array}$ & 2.48 -fold $(1.73 ; 3.75)$ & \\
\hline Proportional error (\%) & $17.4 \%(12.2 ; 21.2)$ & \\
\hline Additive error (mg/l) & $0.011(0.004 ; 0.017)$ & \\
\hline \multicolumn{3}{|c|}{$\begin{array}{l}{ }^{\dagger} \text { Intrinsic clearance of moxifloxacin when given at steady state within rifampicin-based TB treatment and no efavire } \\
\text { All clearance and volume parameters have been allometrically scaled with fat-free mass, and the typical values rep } \\
{ }^{\text {"Pl }} \text { - empirical } 95 \% \mathrm{Cl} \text { obtained with a nonparametric bootstrap }(\mathrm{n}=300) \text {. } \\
{ }^{\text {"P The }} \text { These scalic bioavailability is the fraction of the drug, which is absorbed, crosses the gut wall without any change, } \\
\text { prior These parameters were estimated using a prior, as detailed in text of the original analysis [29]. } \\
\text { ART: Antiretroviral therapy; BSV: Between-subject variability; BOV: Between-occasion variability; TB: Tuberculosis. }\end{array}$} \\
\hline
\end{tabular}

moxifloxacin concentrations in this patient cohort [31], which are further decreased by up to $30 \%$ [8,31] as a result of drug interactions with efavirenz-based ART and rifampicin - a known inducer of UGT and P-glycoprotein [35]. After adjusting for these and other covariates, some of the unexplained between subject variability in moxifloxacin PK may be attributed to genetic variability. 


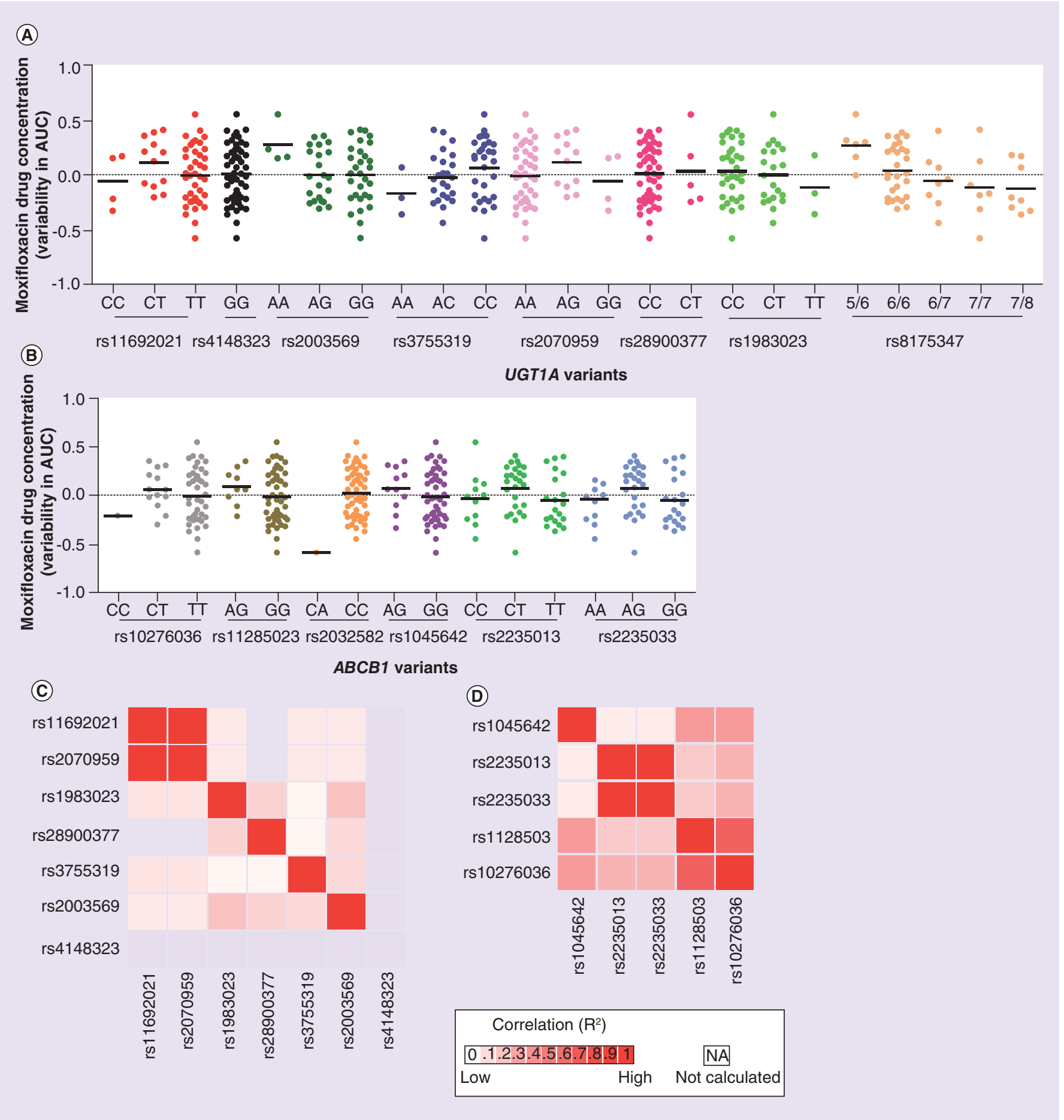

Figure 1. Distribution of moxifloxacin AUC and genotypes/linkage disequilibrium plots. Distributions of (A) UGT1A and (B) ABCB1 genotypes using variability in mean moxifloxacin concentration (AUC) $\mu \mathrm{g} \cdot \mathrm{h} / \mathrm{ml}$ from the base nonlinear mixed-effects model, and linkage disequilibrium between (C) UGT1A (D) and $A B C B 1$ SNPs.

More than 113 different $U G T 1 A 1$ variants have been described, which can confer reduced or increased activity as well as inactive or normal enzymatic phenotypes $[18,20]$. The UGT1A1 rs8175347 SNP in the gene promoter region covering the TATA box consists of variation in a short $(T A ; n)$ sequence from five to eight TA repeats. This SNP has been extensively studied for association between the UGT1A1*28 (TA7) allele and toxicity related 


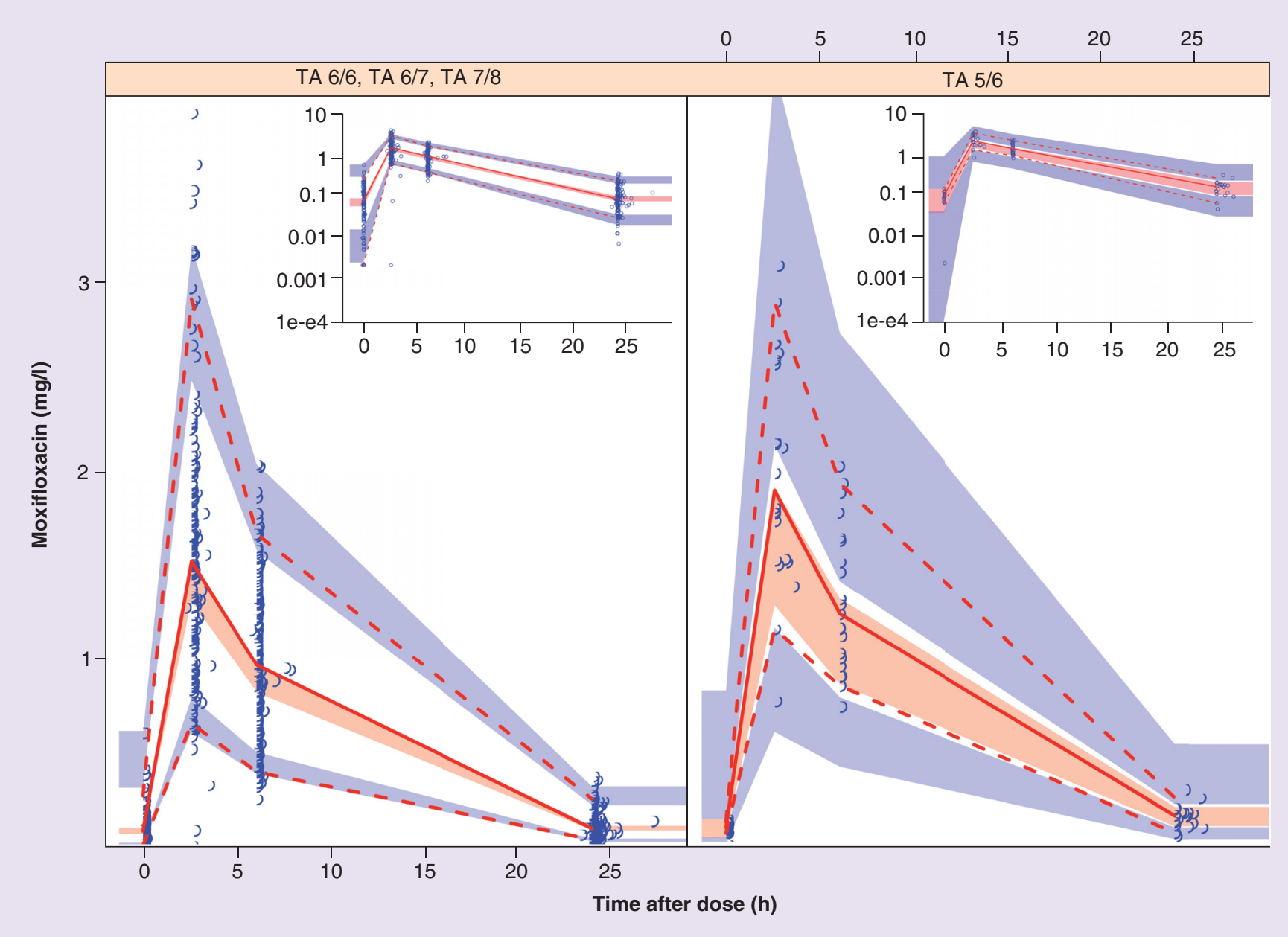

Figure 2. Visual predictive checks stratified by genotype of rs8175347. The dashed and solid lines are the 5th, median and 95 th percentiles of the observed concentrations, while the shaded regions represent the corresponding $95 \% \mathrm{Cls}$ for the same percentiles. The subplot in each stratum shows the same visual predictive checks with a logarithmic transformation applied to the $y$-axis.

to the cancer drug irinotecan $[18,36$. The TA6 and TA7 variants are frequently seen in most populations studied, while the TA5 (UGT1A1*36) and TA8 $\left(U G T 1 A 1^{*} 37\right)$ are found almost exclusively in African populations; TA6 $\left(U G T 1 A 1^{*} 1\right)$ being the wild-type $[18,37]$. Frequencies of the TA repeats observed in our cohort were similar to those previously reported in South African patients; however, the frequency of the TA6 allele was higher $(0.63$ vs 0.5$)$ and TA7 lower ( 0.23 vs 0.37$)$ in our cohort compared with a previous published black South African cohort [37]. Homozygotes for TA7 $\left(U G T 1 A 1^{*} 28 /{ }^{*} 28\right)$ and less commonly the TA8 genotype have been linked to decreased transcriptional activity and lower activity of the gene compared with the wild-type, resulting in decreased glucuronidation of the toxic SN-38 metabolite of irinotecan. The TA7 and TA8 containing genotypes are, therefore, expected to result in decreased metabolism, lower clearance, higher bioavailability and drug exposure as seen with thyroxine (T4) [38]. These and other studies suggest that an increased number of TA repeats may be associated with higher drug exposure. However, we demonstrate higher moxifloxacin concentrations with heterozygous TA5/6 genotypes compared with TA6/6, TA6/7 and TA7/8 genotypes. Similar findings were reported with telmisartan [39] raloxifene [40] and moxifloxacin in healthy individuals [19], where higher concentrations of metabolites and resultant lower drug exposures were observed with TA7 $\left(U G T 1 A 1{ }^{*} 28\right)$. The reasons for this contrasting effect are not entirely clear, but may be linked to extensive sharing of exons in the UGT1A family and genetic linkage between many UGT variants (which may differ between ethnic groups) contributing to discordance between findings. Furthermore, uncertainty exists on the extent of functional activity of the UGT variants studied. 
We report effects of the AC and AA variants in the rs3755319 UGT1A SNP resulting in higher clearance (CL) and decreased moxifloxacin exposures; however, these effects were not robust enough to be included in the final NLME model. Previous studies have reported nonsignificant effects of this SNP on exposure to other drugs such as ABT-751 used to treat patients with neoplasms [41]. No significant effects of $U G T$ haplotypes were observed in our study.

We found very limited effects of genotypes in the $A B C B 1$ SNPs studied, with the exception of rs 2032582 where we found $40 \%$ reduced prehepatic bioavailability associated with lower moxifloxacin concentrations in the presence of the CA genotype. Similar effects of this variant have been reported previously [23]. However, we had only one individual in this genotype group, likely as a result of our limited sample size. A previous study testing the effect of the rs2032582 SNP on efavirenz concentrations in South African patients found limited effects on drug exposure [42]. We found no significant effects of $A B C B 1$ haplotypes in our study. The $A B C B 1$ variants $1236 \mathrm{C}>\mathrm{T}$ (rs1128503), 2677G $>$ T/A (rs2032582) and 3435C > T (rs1045642) occur at high-allele frequencies and create a common haplotype; therefore, they have been widely studied in relation to a number of drug classes [23]. A recent review investigating the effects of these SNPs on drug exposure and PK parameters in over 300 studies concluded that although these had significant effects on drug disposition and response, findings were in part conflicting and the clinical implications limited [23].

We acknowledge the limitations of our study. This includes a relatively small sample of patients with drug concentration data, and the lack of significant associations observed between SNPs studied and PK parameters for moxifloxacin may be due to inadequate numbers of patients in the groups for each of the genotypes studied. The concentrations of moxifloxacin metabolites M1 and M2 were not measured and we are unable to draw any conclusions on the effects of the genetic variation in UGT on moxifloxacin glucuronidation to its inactive metabolites. We included a limited number of SNPs in our study based on previous literature that showed association between these SNPs and drug response, toxicity or disease susceptibility. Other relevant $U G T 1 A$ and $A B C B 1$ SNPs that were not included in this study may significantly impact moxifloxacin PK parameters. Genotype frequencies in our population may differ from other populations and our findings may therefore be limited to similar African populations. The PK data were collected with sparse sampling. While NLME modeling has been shown to be suitable to analyze sparse data, our model had sometimes limited capability to separate effect on $\mathrm{CL}_{\text {int }}$ versus prehepatic bioavailability, parameters which both affect total exposure, as seen with TA5/6 repeat in rs 8175347 . In such cases, the most statistically significant effect was included in the model. We previously reported significant effect of efavirenz on moxifloxacin pharmacokinetics (42\% increased clearance and resultant 30\% lower AUC) due to induction of the UGT enzyme in these patients. While the NLME model adjusted for the effect of efavirenz on moxifloxacin PK and should be able to separate this from the genotype effect it is possible that if efavirenz induction mimics the effects of genetic variants on enzyme activity or if mutations in the UGT enzyme binding sites alter the extent of efavirenz induction, that this may interfere with the model's ability to detect the effect of the genotypes or differentiate between these effects.

\section{Conclusion}

We have observed increased prevalence of genetic variability in $U G T 1 A$ and $A B C B 1$ in our cohort of South African patients. Genotypes of UGT1A SNPs (rs8175347) and to a lesser extent $U G T 1 A$ (rs3755319) and $A B C B 1$ (rs2032582) were associated with significant differences in moxifloxacin drug concentrations or PK parameters. The clinical relevance of these changes in moxifloxacin drug exposure on TB treatment outcomes needs further investigation, although given that moxifloxacin exhibits concentration-dependent bactericidal activity, changes in drug concentrations are likely to impact efficacy.

PK-PD targets including AUC: MIC ratios $>53$ or 100 have been suggested to be associated with improved outcomes and reduced risk of emergent drug resistance. Moxifloxacin drug concentrations in our study were low overall. It is therefore plausible that SNPs associated with lower or higher drug concentrations may affect individual patient's likelihood of achieving these targets.

\section{Acknowledgements}

The authors gratefully acknowledge the study participants for their participation in the IMPRESS pharmacokinetic study; study nurses, counsellors, clinicians, the study co-ordinator D Govender and the IMPRESS study team for their work in the pharmacokinetic study. Special acknowledgement to M Govender and the pharmacy team for support on the study. We thank the following laboratories and laboratory staff; CAPRISA laboratory (N Samsunder, and lab staff), KwaZulu-Natal Research Institute for Tuber- 
culosis and HIV (KRITH) Pharmacology Core and Medical Microbiology Laboratories and Global Clinical \& Viral Laboratories, for laboratory support. We thank D Munatsi and P Radebe for data management on the study, and J Bredenkamp (Forest Molecular Genetics Programme, University of Pretoria) for the TaqMan ${ }^{\circledR}$ OpenArray genotyping assay analysis. The Division of Clinical Pharmacology at the University of Cape Town, acknowledges Novartis Pharma for their support of the development of pharmacometric skills in Africa. The sponsor of the study had no role in study design, data collection, data analysis, data interpretation or writing of the report. All authors had access to data, commented on drafts and approved the final report. The corresponding author had final responsibility for the decision to submit for publication.

\section{Author contributions}

The study concept and design were done by A Naidoo, N Padayatchi, K Naidoo, H Mcllleron, MS Pepper and V Ramsuran. Drafting of the manuscript was done by A Naidoo, V Ramsuran, P Denti and M Chaudhry. Statistical analysis: NLME modeling and data analysis were done by P Denti and M Chaudhry. General statistical support was given by N Yende-Zuma. Acquisition, analysis or interpretation of data and critical revision of the manuscript for important intellectual content were done by all the authors.

\section{Financial \& competing interests disclosure}

This publication was made possible by grant no. 5R24TW008863 from the Office of Global AIDS Coordinator and the US Department of Health and Human Services, NIH (NIH Office of AIDS Research [OAR] and NIH Office of World AIDS Research [OWAR]). Research reported in this publication was supported by the European \& Developing Countries Clinical Trials Partnership (EDCTP; TA.2011.40200.044), the South African Medical Research Council CAPRISA HIV-TB Pathogenesis and Treatment Research Unit the South African Medical Research Council under a self-initiated research grant. Study drug was donated by Bayer Pharmaceuticals. A Naidoo was the recipient of the University of KwaZulu-Natal College of Health Sciences Scholarship. H Mclleron is supported in part by the National Research Foundation (NRF) of South Africa (grant no. 90729). K Naidoo and N Padayatchi were supported by the Columbia University-South Africa Fogarty AIDS260 International Training and Research Program (AITRP, grant no. D43TW000231). MS Pepper and M Chaudhry are supported by the University of Pretoria's Institute for Cellular and molecular medicine and the South African Medical Research Council (University Flagship and Stem Cell EMU awards). V Ramsuran was supported in part by a research Flagship grant from the South African Medical Research Council (MRC-RFA-UFSP-01-2013/UKZN HIVEPI). The contents of the manuscript are solely the responsibility of the authors and do not necessarily represent the official views of the US government, EDCTP, NRF, Fogarty International Center, NIH or the Medical Research Council. N Padayatchi is the principal study investigator, on the Improving Retreatment Success Trial (IMPRESS; NCT02114684). Bayer Pharmaceuticals donated the study drug (moxifloxacin) used during the trial. The authors have no other relevant affiliations or financial involvement with any organization or entity with a financial interest in or financial conflict with the subject matter or materials discussed in the manuscript apart from those disclosed.

No writing assistance was utilized in the production of this manuscript.

Summary points

- Moxifloxacin is emerging as a key drug in the treatment of drug-susceptible and drug-resistant tuberculosis.

- Moxifloxacin is metabolized by UGT enzymes and is a substrate of P-glycoprotein, coded by UGT and the $A B C B 1$ gene.

- Genes encoding for UGT and $A B C B 1$ enzymes, are known to be highly polymorphic leading to altered concentrations of other drugs and of moxifloxacin in healthy individuals.

- We report in this study the prevalence of genetic variation in UGT1A and ABCB1 genes in black South African tuberculosis patients and assess effect of these on moxifloxacin pharmacokinetics.

- Genotypes for selected UGT1A and ABCB1 SNPs were determined using a TaqMan ${ }^{\circledR}$ Genotyping OpenArray ${ }^{\mathrm{TM}}$ and high-resolution melt analysis for rs8175347.

- A nonlinear mixed-effects model was used to describe moxifloxacin pharmacokinetics.

- UGT1A SNPs rs8175347 and rs3755319, and ABCB1 SNP rs2032582 were significantly associated with changes to moxifloxacin pharmacokinetic parameters and drug exposure.

- Genetic variability in UGT1A and $A B C B 1$ contributes to the wide interindividual variability reported in moxifloxacin pharmacokinetics in the context of tuberculosis treatment.

- Although the clinical relevance of the effects of genetic variation on moxifloxacin pharmacokinetic requires further investigation moxifloxacin exhibits concentration-dependent activity and hence changes to drug exposures are likely to impact clinical outcomes. 


\section{References}

Papers of special note have been highlighted as: $\bullet$ of interest; $\bullet \bullet$ of considerable interest

1. Bayer HealthCare AG. Moxifloxacin investigator brochure version 14.0. Bayer HealthCare, Germany (2011).

2. WHO. Global Tuberculosis Report 2016. www.who.int/tb/publications/global_report/en/

3. Dawson R, Diacon AH, Everitt D et al. Efficiency and safety of the combination of moxifloxacin, pretomanid (PA-824), and pyrazinamide during the first 8 weeks of antituberculosis treatment: a Phase $2 \mathrm{~b}$, open-label, partly randomised trial in patients with drug-susceptible or drug-resistant pulmonary tuberculosis. Lancet 385(9979), 1738-1747 (2015).

4. Moodley R, Godec TR. Short-course treatment for multidrug-resistant tuberculosis: the STREAM trials. Eur. Respir. Rev. 25(139), 29-35 (2016).

5. Gumbo T, Louie A, Deziel MR, Parsons LM, Salfinger M, Drusano GL. Selection of a moxifloxacin dose that suppresses drug resistance in Mycobacterium tuberculosis, by use of an in vitro pharmacodynamic infection model and mathematical modeling. J. Infect. Dis. 190(9), 1642-1651 (2004).

6. MacGowan AP. Moxifloxacin (Bay 12-8039): a new methoxy quinolone antibacterial. Expert Opin. Investigational Drugs 8(2), 181-199 (1999).

7. Nijland HM, Ruslami R, Suroto AJ et al. Rifampicin reduces plasma concentrations of moxifloxacin in patients with tuberculosis. Clin. Infect. Dis. 45(8), 1001-1007 (2007).

8. Weiner M, Burman W, Luo CC et al. Effects of rifampin and multidrug resistance gene polymorphism on concentrations of moxifloxacin. Antimicrob. Agents Chemother. 51(8), 2861-2866 (2007).

9. Alffenaar JW, de Vries PM, Luijckx GJ, van Soolingen D, van der Werf TS, van Altena R. Plasma and cerebrospinal fluid pharmacokinetics of moxifloxacin in a patient with tuberculous meningitis. Antimicrob. Agents Chemother. 52(6), 2293-2295 (2008).

10. Pranger AD, Kosterink JG, van Altena R et al. Limited-sampling strategies for therapeutic drug monitoring of moxifloxacin in patients with tuberculosis. Ther. Drug Monit. 33(3), 350-354 (2011).

11. Naidoo A, Naidoo K, McIlleron H, Essack S, Padayatchi N. A review of moxifloxacin for the treatment of drug-susceptible tuberculosis. J. Clin. Pharmacol. 57(11), 1369-1389 (2017).

12. Ramachandran G, Swaminathan S. Role of pharmacogenomics in the treatment of tuberculosis: a review. Pharmgenomics Pers. Med. 5 , 89-98 (2012).

13. Mboowa G. Genetics of sub-Saharan African human population: implications for HIV/AIDS, tuberculosis, and malaria. Int. J. Evol. Biol. 2014, 108291 (2014).

14. Chigutsa E, Visser ME, Swart EC et al. The SLCO1B1 rs 4149032 polymorphism is highly prevalent in South Africans and is associated with reduced rifampin concentrations: dosing implications. Antimicrob. Agents Chemother. 55(9), 4122-4127 (2011).

15. Gengiah TN, Botha JH, Soowamber D, Naidoo K, Abdool Karim SS. Low rifampicin concentrations in tuberculosis patients with HIV infection. J. Infect. Dev. Ctries 8(8), 987-993 (2014).

16. McIlleron $\mathrm{H}$, Willemse $\mathrm{M}$, Werely CJ et al. Isoniazid plasma concentrations in a cohort of South African children with tuberculosis: implications for international pediatric dosing guidelines. Clin. Infect. Dis. 48(11), 1547-1553 (2009).

17. Moise PA, Birmingham MC, Schentag JJ. Pharmacokinetics and metabolism of moxifloxacin. Drugs Today 36(4), 229-244 (2000).

18. Barbarino JM, Haidar CE, Klein TE, Altman RB. PharmGKB summary: very important pharmacogene information for UGT1A1. Pharmacogenet. Genomics 24(3), 177-183 (2014).

19. Hasunuma $\mathrm{T}$, Tohkin $\mathrm{M}$, Kaniwa $\mathrm{N}$ et al. Absence of ethnic differences in the pharmacokinetics of moxifloxacin, simvastatin, and meloxicam among three east Asian populations and Caucasians. Br. J. Clin. Pharmacol. 81(6), 1078-1090 (2016).

20. Stingl JC, Bartels H, Viviani R, Lehmann ML, Brockmoller J. Relevance of UDP-glucuronosyltransferase polymorphisms for drug dosing: a quantitative systematic review. Pharmacol. Ther. 141(1), 92-116 (2014).

21. Ito T, Yano I, Tanaka K, Inui KI. Transport of quinolone antibacterial drugs by human P-glycoprotein expressed in a kidney epithelial cell line, LLC-PK1. J. Pharmacol. Exp. Ther. 282(2), 955-960 (1997).

22. Fromm MF. The influence of $M D R 1$ polymorphisms on P-glycoprotein expression and function in humans. Adv. Drug Deliv. Rev. 54(10), 1295-1310 (2002).

23. Wolking S, Schaeffeler E, Lerche H, Schwab M, Nies AT. Impact of genetic polymorphisms of $A B C B 1$ (MDR1, P-glycoprotein) on drug disposition and potential clinical implications: update of the literature. Clin. Pharmacokinet. 54(7), 709-735 (2015).

24. Hodges LM, Markova SM, Chinn LW et al. Very important pharmacogene summary: ABCB1 (MDR1, P-glycoprotein). Pharmacogenet. Genomics 21(3), 152-161 (2011).

25. FDA. Guidance for industry bioanalytical method validation, Department of Health and Human Services, Food and Drug Administration. www.fda.gov/downloads/Drugs/GuidanceComplianceRegulatoryInformation/Guidances/ucm070107.pdf

26. Whirl-Carrillo M, McDonagh EM, Hebert JM et al. Pharmacogenomics knowledge for personalized medicine. Clin. Pharmacol. Ther. 92(4), 414-417 (2012). 
27. Farrar JS, Palais RA, Wittwer CT. Snapback primer genotyping of the Gilbert syndrome UGT1A1 (TA)(n) promoter polymorphism by high-resolution melting. Clin. Chem. 57(9), 1303-1310 (2011).

28. Machiela MJ, Chanock SJ. LDlink: a web-based application for exploring population-specific haplotype structure and linking correlated alleles of possible functional variants. Bioinformatics 31(21), 3555-3557 (2015).

29. Beal S, Sheiner L, Boeckmann A, Bauer R. NONMEM users guides (1989-2009). ICON Development Solutions MD, USA (2009).

30. Keizer RJ, Karlsson MO, Hooker A. Modeling and simulation workbench for NONMEM: tutorial on Pirana, PsN, and Xpose. CPT: Pharmacometrics Systems Pharmacology 2, e50 (2013).

31. Naidoo A, Chirehwa M, McIlleron $\mathrm{H}$ et al. Effect of rifampicin and efavirenz on moxifloxacin concentrations when co-administered in patients with drug-susceptible TB. J. Antimicrob. Chemother. 72(5), 1441-1449 (2017).

32. Janmahasatian S, Duffull SB, Ash S, Ward LC, Byrne NM, Green B. Quantification of lean bodyweight. Clin. Pharmacokinet. 44(10), 1051-1065 (2005).

33. Peloquin CA, Hadad DJ, Molino LP et al. Population pharmacokinetics of levofloxacin, gatifloxacin, and moxifloxacin in adults with pulmonary tuberculosis. Antimicrob. Agents Chemother. 52(3), 852-857 (2008).

34. Pranger AD, van Altena R, Aarnoutse RE et al. Evaluation of moxifloxacin for the treatment of tuberculosis: 3 years of experience. Eur. Respir. J. 38(4), 888-894 (2011).

35. Kim KA, Park PW, Liu KH et al. Effect of rifampin, an inducer of CYP3A and P-glycoprotein, on the pharmacokinetics of risperidone. J. Clin. Pharmacol. 48(1), 66-72 (2008).

36. Minami H, Sai K, Saeki M et al. Irinotecan pharmacokinetics/pharmacodynamics and UGT1A genetic polymorphisms in Japanese: roles of $U G T 1 A 1^{*} 6$ and ${ }^{*} 28$. Pharmacogenet. Genomics 17(7), 497-504 (2007).

37. Horsfall LJ, Zeitlyn D, Tarekegn A et al. Prevalence of clinically relevant UGT1A alleles and haplotypes in African populations. Annals Human Genet. 75(2), 236-246 (2011).

38. Santoro AB, Vargens DD, Barros Filho Mde C et al. Effect of UGT1A1, UGT1A3, DIO1 and DIO2 polymorphisms on L-thyroxine doses required for TSH suppression in patients with differentiated thyroid cancer. Br. J. Clin. Pharmacol. 78(5), 1067-1075 (2014).

39. Shin HJ, Kim JE, Lim SJ et al. Effect of UGT1A1 and UGT1A3 polymorphisms on pharmacokinetics of telmisartan in Korean. Clin. Ther. 37(8), e87 (2015).

40. Trontelj J, Marc J, Zavratnik A, Bogataj M, Mrhar A. Effects of $U G T 1 A 1 * 28$ polymorphism on raloxifene pharmacokinetics and pharmacodynamics. Br. J. Clin. Pharmacol. 67(4), 437-444 (2009).

41. Innocenti F, Ramirez J, Obel J et al. Preclinical discovery of candidate genes to guide pharmacogenetics during Phase I development: the example of the novel anticancer agent ABT-751. Pharmacogenet. Genomics 23(7), 374-381 (2013).

42. Swart M, Ren Y, Smith P, Dandara C. ABCB1 4036A $>$ G and 1236C $>$ T polymorphisms affect plasma efavirenz levels in South African HIV/AIDS patients. Front. Genet. 3, 236 (2012). 